EPJ Web of Conferences 41, 09003 (2013)

DOI: 10.1051/epjconf/20134109003

(C) Owned by the authors, published by EDP Sciences, 2013

\title{
Self-phase modulation of a single-cycle THz pulse
}

\author{
D. Turchinovich ${ }^{1,2}$, J. M. Hvam², and M. C. Hoffmann ${ }^{3}$
}

${ }^{1}$ Max Planck Institute for Polymer Research, Ackermannweg 10, 55128 Mainz, Germany

${ }^{2}$ DTU Fotonik, Technical University of Denmark, Ørsteds Plads 343, 2800 Lyngby, Denmark

${ }^{3}$ SLAC Linear Accelerator Laboratory, 2575 Sand Hill Road, Menlo Park, CA, U.S.A.

\begin{abstract}
We demonstrate self-phase modulation (SPM) of a single-cycle $\mathrm{THz}$ pulse in a semiconductor, using bulk $n$-GaAs as a model system. The SPM arises from the heating of free electrons in the electric field of the $\mathrm{THz}$ pulse. Electron heating leads to an ultrafast reduction of the plasma frequency, which results in a strong modification of the THz-range dielectric function of the material. THz SPM is observed directly in the time domain as a characteristic reshaping of single-cycle $\mathrm{THz}$ pulse. In the frequency domain, it corresponds to a strong frequency-dependent refractive index nonlinearity of $n$-GaAs, which is found to be both positive and negative within the broad spectrum of the $\mathrm{THz}$ pulse. The spectral position of zero nonlinearity is defined by the electron momentum relaxation rate. Nonlinear spectral broadening and compression of the single-cycle THz pulse was also observed.
\end{abstract}

Self-phase modulation (SPM), a fundamental nonlinear-optical effect, leads to intensitydependent temporal and spectral reshaping of optical signals as they propagate through a nonlinear medium. Here we demonstrate the SPM in a new mode - at $\mathrm{THz}$ frequencies, using a doped semiconductor, $n$-GaAs, as an efficient nonlinear medium. However, any other semiconductor with complex band structure will also show SPM along the lines described in this work. Further, we demonstrate the SPM occurring in a single-cycle regime, with the signal spectrum covering many octaves of frequencies. As will be shown below, single-cycle $\mathrm{THz}$ pulses can be used as accessible model tools for direct observation of general nonlinear optical effects occurring in the single-cycle regime, and the findings obtained in the $\mathrm{THz}$ range can be generalized to other spectral ranges.

The THz-range optical nonlinearity of a semiconductor, such as $n$-GaAs used in this work, arises from the nonlinear response of the free-carrier plasma to the $\mathrm{THz}$ ponderomotive excitation. Semiconductors with free carriers are good absorbers of $\mathrm{THz}$ radiation, and their $\mathrm{THz}-$ range complex-valued dielectric function $\hat{\varepsilon}$ is well described by the basic Drude plasma model [1,2]

$$
\hat{\varepsilon}(\omega)=(n+i \alpha c / 2 \omega)^{2}=\varepsilon_{d c}-\omega_{p}^{2} /\left(\omega^{2}-i \omega / \tau_{r}\right)
$$

or one of its extensions. Here $n$ and $\alpha$ are the frequency-dependent refractive index and power absorption coefficient, respectively, $\tau_{\mathrm{r}}$ is a carrier momentum relaxation time, and $\varepsilon_{d c}$ is the background "static" dielectric constant of semiconductor in the absence of free carriers (ignoring, for simplicity, the dispersion due to a phonon contribution to $\left.\varepsilon_{d c}\right) . \omega_{p}=\left(N e^{2} / \epsilon_{0} m\right)^{1 / 2}$ is the plasma

This is an Open Access article distributed under the terms of the Creative Commons Attribution License 2.0, which permits unrestricted use, distribution, and reproduction in any medium, provided the original work is properly cited. 
frequency, where $e$ is the elementary charge, $N$ is the free carrier density, $\epsilon_{0}$ is the vacuum permittivity, and $m$ is the carrier effective mass.

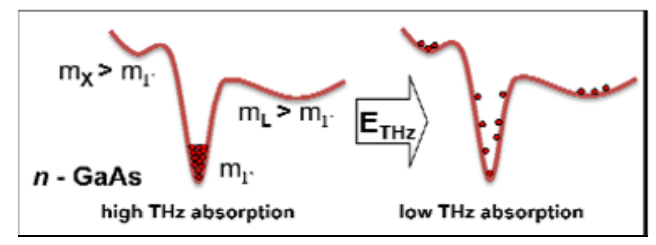

Fig. 1. Mechanism of THz nonlinearity in doped semiconductors by carrier heating in $\mathrm{THz}$ field.

It directly follows from eq. (1) that a change in plasma frequency $\omega_{p}$ resulting from the $\mathrm{THz}$ light - matter interaction will lead to a change in the THz absorption $\alpha$ and the refractive index $n$ of the semiconductor, i.e. to nonlinear absorption and SPM. In a doped semiconductor where the freecarrier density is fixed, the change in the plasma frequency can be introduced by the manipulation of the carrier effective mass. In our work we used the ponderomotive THz excitation of the loweffective-mass free electrons, initially occupying the bottom of the $\Gamma$-valley of GaAs, into the states with increased effective mass, such as the satellite valleys. Such THz-induced electron heating leads to a near-instantaneous decrease of electron mobility and to a drop in $\mathrm{THz}$ absorption $[3,4]$, as shown in Fig. 1. As a sample we used an $n$-GaAs crystal with the thickness $d$ $=0.4 \mathrm{~mm}, N=510^{15} \mathrm{~cm}^{-3}$, and $\tau_{\mathrm{r}}=94 \mathrm{fs}$.

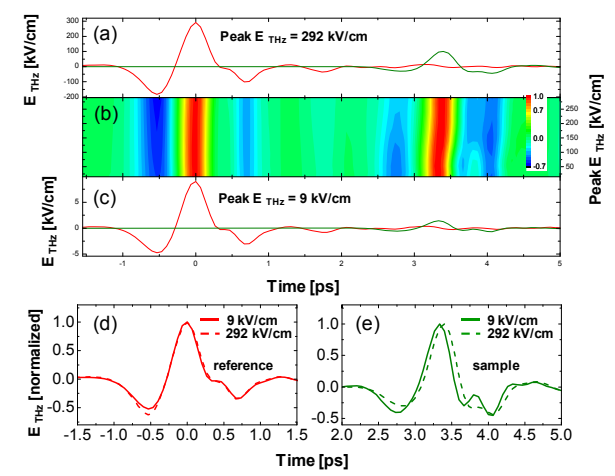

Fig. 2. $\mathrm{THz}$ SPM in $\mathrm{n}-\mathrm{GaAs}$ in the time domain. Peak $\mathrm{E}_{\mathrm{THz}}$ of reference $\mathrm{THz}$ pulse in (a) is 292 $\mathrm{kV} / \mathrm{cm}$, and in (c) is $9 \mathrm{kV} / \mathrm{cm}$. (b) Reference and sample pulses normalized to their maxima, for the whole range of peak $E_{T H z}$ of $9-292 \mathrm{kV} / \mathrm{cm}$. (d,e) Normalized reference (d) and sample (e) pulses for the cases of peak $E_{T H z}$ of $9 \mathrm{kV} / \mathrm{cm}$ and $292 \mathrm{kV} / \mathrm{cm}$, demonstrating the SPM.

We demonstrated the THz SPM using nonlinear THz time-domain spectroscopy (NL THz TDS) [4] on our sample in transmission configuration. The peak field strength of the THz pulse $E_{T H z}$ in the range $9-292 \mathrm{kV} / \mathrm{cm}$ was controlled by a pair of crossed wire-grid polarizers placed after the strong-field $\mathrm{THz}$ emitter [4,5]. For each peak $E_{T H z}$ two $\mathrm{THz}$ pulses were recorded: a reference pulse propagating through the beam path in a spectrometer in vacuum, and a sample pulse propagated through the same geometrical path, but now with the sample inserted into it. Both reference and sample pulses were recorded in the same reference time frame, which allowed us to extract the spectral phase acquired by the $\mathrm{THz}$ pulse in the sample, for each value of peak $E_{T H z}$ used in the experiments.

The results of these measurements are presented in Fig. 2. The reference and sample THz pulses are centered at the time delays of 0 and 3.3 ps, respectively. In Figs. 2 (a,c) we show the raw data for the case of the weakest and the strongest $\mathrm{THz}$ pulses with peak $E_{T H z}$ of $9 \mathrm{kV} / \mathrm{cm}$ and 292 $\mathrm{kV} / \mathrm{cm}$. Clear saturable absorption is observed [4], as explained by the mechanism in Fig. 1. In Fig. 2 (b) the reference and sample $\mathrm{THz}$ pulses, normalized to their respective maxima, are shown. A clear time shift of the sample $\mathrm{THz}$ pulse with increase in peak $E_{T H z}$ is observed, manifesting the SPM in time domain. In Figs. $2(\mathrm{~d}, \mathrm{e})$ the normalized reference and sample $\mathrm{THz}$ 
pulses for the extreme values of $E_{T H z}$ in our measurements are shown. The very small reshaping of reference pulses, not demonstrating any temporal shift, is due to the non-ideality of wire-grid polarizers. The significant time shift of the sample THz pulses is due to SPM in $n$-GaAs.
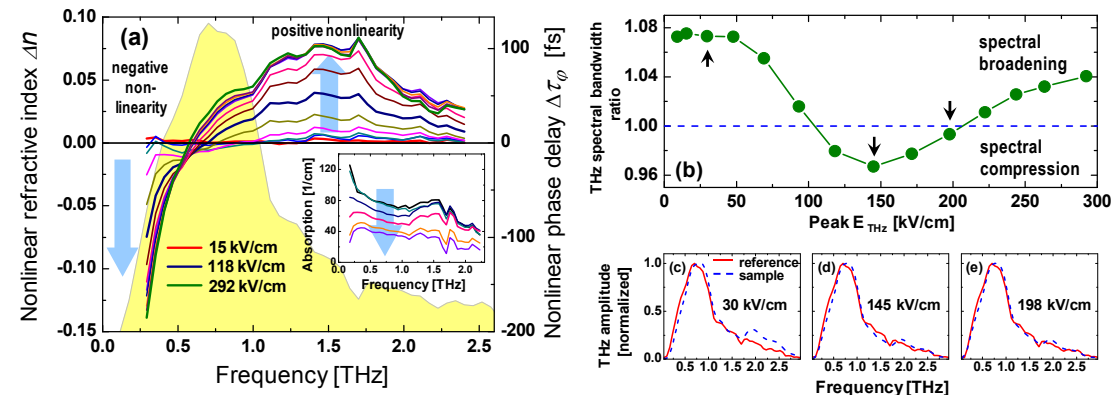

Fig. 3. (a) THz SPM in n-GaAs in spectral domain. Nonlinear contribution to refractive index $\Delta n$, and corresponding nonlinear phase delay as a function of frequency and peak $E_{T H z}$ in the range 9 $292 \mathrm{kV} / \mathrm{cm}$. Blue arrows show the positive and negative refractive index nonlinearity with increasing peak $E_{T H z}$. The amplitude spectrum of the reference $\mathrm{THz}$ pulse is shown as a background. (b-e) Nonlinear spectral broadening and compression of a THz pulse in $n$-GaAs. (b)The ratio of effective bandwidths of the sample and reference pulses, as a function of peak $E_{T H z}$. (c-e) Examples of spectral dynamics: normalized amplitude spectra of reference and sample $\mathrm{THz}$ pulses measured at peak $\mathrm{THz}$ field values of $30 \mathrm{kV} / \mathrm{cm}, 145 \mathrm{kV} / \mathrm{cm}$, and $198 \mathrm{kV} / \mathrm{cm}$.

In Fig. 3 (a) we demonstrate the nonlinear modification of a frequency-dependent refractive index of $n$-GaAs, as a correction to the refractive index of $n$-GaAs experienced by the weakest $\mathrm{THz}$ pulse in our experiments with peak $\mathrm{E}_{\mathrm{THz}}=9 \mathrm{kV} / \mathrm{cm}$. The corresponding phase delay is also indicated in this figure. An amplitude spectrum of a reference $\mathrm{THz}$ pulse is shown in the background. Interestingly, but not quite unexpectedly, given the ultra-broadband nature of a single-cycle pulse, the refractive index nonlinearity is found to be both positive (at higher frequencies) and negative (at lower frequencies) within the bandwidth of a single-cycle $\mathrm{THz}$ pulse. This behavior directly follows from the Drude model (eq. 1), and the crossing point between the positive and negative nonlinearity trends is determined by (but not equal to) the electron momentum relaxation rate $\tau_{\mathrm{r}}[7]$.

In Figs. 3 (b-e) we show yet another effect typical for the propagation of ultrashort optical signals in nonlinear systems, and associated with SPM (see e.g. [6]) - the nonlinear spectral broadening and compression of the THz pulse in n-GaAs, depending on peak $E_{T H z}$.

The detailed investigation of THz SPM in semiconductors is presented in [7]. The movie visualising the nonlinear absorption, SPM, and spectral dynamics of single-cycle THz pulses in $n$ GaAs can be found in [8].

1. M. Dressel and G. Grüner, Electrodynamics of solids: Optical properties of electrons in matter, (Cambridge Univ. Press, Cambridge, 2002).

2. R. Huber, F. Tauser, A. Brodschelm, M. Bichler, G. Abstreiter, and A. Leitenstorfer, Nature 414, 286 (2001).

3. M. C. Hoffmann, J. Hebling, H. Y. Hwang, K.-L. Yeh, and K. A. Nelson, J. Opt. Soc. Am. B 26, A29 (2009).

4. M. C. Hoffmann and D. Turchinovich, Appl. Phys. Lett. 96, 151110 (2010).

5. M. C. Hoffmann, B. S. Monozon, D. A. Livshits, E. U. Rafailov, and D. Turchinovich, Appl. Phys. Lett. 97, 231108 (2010).

6. X. Liu, J. Lægsgaard, and D. Turchinovich, Opt. Express 18, 15475 (2010).

7. D. Turchinovich, J. M. Hvam, and M. C. Hoffmann, Phys. Rev. B 85, 201304(R) (2012).

8. Movie: http://www.ultrafast.dk/movies/nGaAs.htm 